\title{
Comparison of rapid automated laser fluorescence analysis of DNA fingerprints with four other computer-assisted approaches for studying relationships between Acinetobacter baumannii isolates
}

\author{
C. A. WEBSTER, K. J. TOWNER $\dagger$, H. HUMPHREYS, B. EHRENSTEIN*, D. HARTUNG* and \\ H. GRUNDMANN*
}

Department of Microbiology and PHLS Laboratory, University Hospital, Queen's Medical Centre, Nottingham NG7 2UH and *Institut für Umweltmedizin und Krankenhaushygiene, Universitätsklinik Freiburg, 79106 Freiburg, Germany

\begin{abstract}
The relationships between isolates suggested by a novel DNA typing method (RAPDALFA) that combines randomly amplified polymorphic DNA with automated on-line laser fluorescence analysis of DNA fragments were compared with those suggested by four other computer-assisted typing strategies (biotyping, antibiogram typing, pulsedfield gel analysis of chromosomal fingerprints and arbitrarily-primed DNA amplification with three different primers) for 25 isolates of Acinetobacter baumannii obtained from 12 different hospitals in four countries over a period of 12 years. The results obtained by cluster analysis with two different software packages confirmed that the relationships suggested by RAPD-ALFA were robust and essentially similar to those suggested by the other more laborious computer-assisted typing methods. The technique of RAPD-ALFA appears to offer the possibility of routine on-line molecular identification and typing of isolates from particular hospital wards or units (e.g., intensive care units), and could, therefore, play a key role in the early recognition and prevention of outbreaks of infection.
\end{abstract}

\section{Introduction}

Rapid DNA fingerprinting analysis methods based on the functionally interchangeable methods of arbitrarily primed amplification of chromosomal DNA (AP-PCR) $[1,2]$ and randomly amplified polymorphic DNA (RAPD) [2,3] are being used increasingly in many microbiology laboratories for epidemiological typing of an ever-increasing range of bacteria. However, difficulties arise with these methods when comparing visually the fingerprint banding patterns from a large number of isolates examined on different gels over extended time intervals, while other problems include a reported lack of reproducibility and difficulties with the standardisation of equipment and reagents [4-6]. In an attempt to overcome some of these drawbacks, a novel method combining RAPD analysis with automated laser fluorescence analysis (ALFA) of DNA fragments has been developed in which computerised cluster analysis is used for recognition of related strains within a single working day [7]. This technique (RAPD-

Received 18 Aug. 1995; revised version accepted 29 Sept. 1995.

†Corresponding author: Dr K. J. Towner.
ALFA) achieves high resolution of the RAPD amplification patterns by the use of rapid denaturing sequencing gels that are capable of discriminating DNA products differing by only a single base [8]. The resulting data are digitised and used for strain comparisons and the generation of dendrograms without the loss of information that may occur inadvertently during the scanning of gel images generated by other methods.

Although the RAPD-ALFA technique has been shown to generate reproducible results with single strains and epidemiologically related isolates [7], the extremely precise nature of the resolution means that the technique could provide too much discrimination to recognise links between closely related, but not identical strains. To investigate this possibility, this study compared the strain relationships suggested by RAPD-ALFA with those suggested by four other computer-assisted typing strategies - employing a different software package and clustering algorithm for isolates of Acinetobacter baumannii collected from several geographically separate hospitals. Antibioticresistant strains of $A$. baumannii play a significant role in colonisation and infection of patients admitted to 
hospital intensive care units (ICUs) $[9,10]$. They may be isolated from the hospital environment, from colonised or infected patients, and from the hands of staff [9-12], and as opportunist pathogens they are responsible for a wide range of nosocomial infections, including bacteraemia, pneumonia, urinary tract infection and meningitis [9]. No single typing system has so far gained acceptance for typing Acinetobacter spp., and this area is the subject of ongoing research.

\section{Materials and methods}

\section{Bacteria and reagents}

Twenty-nine Acinetobacter strains isolated over a period of 12 years were obtained from 12 different hospitals in four countries. The 25 strains (Table 1) included in the study were those strains identified originally as $A$. baumannii on the basis of biochemical tests performed on initial isolation at the various hospitals. For the purpose of comparing methods, two geographically related groups of strains are highlighted throughout this paper - strains nos. 1, 4, 5, 6, 7, 23, 24, 25 and 26; and strains nos. 27, 28 and 29 - comprising strains from Nottingham and Wales, respectively (Table 1). The strains were stored at $-70^{\circ} \mathrm{C}$ in Skim Milk Powder (Oxoid L31) 10\% w/v in sterile distilled water and then subcultured, as required, on to CLED Agar (Oxoid) and incubated overnight at $30^{\circ} \mathrm{C}$ immediately before analysis by the RAPD-ALFA method and four other computer-assisted typing strategies. All chemicals and reagents were purchased from Sigma or were as described previously for each individual method listed below, with the exception of PCR reagents (Boehringer Mannheim) and custom-synthesised oligonucleotides (Oswel DNA Service, Edinburgh) for RAPD reactions.

\section{$R A P D-A L F A$}

The RAPD-ALFA procedure involves random-primed amplification with fluorescent primers, followed by online automated laser fluorescence analysis of high resolution DNA fingerprints separated on polyacrylamide sequencing gels [7]. The method used was as described by Grundmann et al. [7], except that two different primers were used: (1) a single primer derived from the core region of bacteriophage M13 (5'GAGGGTGGCGGTTCT-3'); and (2) primer DAF4 (5'-CGGCAGCGCC-3') [13]. Stored fluorograms were analysed with the Windows version of the GelCompar Software Version 3.10 (Applied Maths, Kortrijk, Belgium) after conversion of the ALF data into the GelCompar format. Separate electrophoresis gels were normalised according to the internal standards contained in each track (100 bp, $1064 \mathrm{bp}$ ). Dendrograms for cluster analysis were based on similarity matrices calculated from the Pearson correlation and UPGMA algorithm. All strains were examined in duplicate and comparison was based on amplification products ranging from 100 to $700 \mathrm{bp}$ as previous work [7] has shown an overall reproducibility of $>95 \%$ in similarity coefficients calculated from DNA fragments of $<700 \mathrm{bp}$. Final analysis of the dendrograms generated was based on the $72 \%$ similarity level shown previously [7] to distinguish unambiguously between unrelated strains of $A$. baumannii.

\section{Biotyping}

Based on the recommendations of Bouvet and Grimont [14] and Kämpfer et al. [15], each strain was examined in the following 27 tests: growth with $\beta$-alanine, L-arginine, D-asparagine, L-aspartate, azelate, citraco-

Table 1. Strains of Acinetobacter included in the study

\begin{tabular}{|c|c|c|}
\hline Strain no. & $\begin{array}{l}\text { Patient or } \\
\text { environment }\end{array}$ & Source (year) \\
\hline 1 & Patient & UHN, Nottingham, England (1982) \\
\hline 4 & Patient & UHN, Nottingham, England (1993) \\
\hline 5 & Patient & UHN, Nottingham, England (1993) \\
\hline 6 & Environment & UHN, Nottingham, England (1993) \\
\hline 7 & Environment & UHN, Nottingham, England (1993) \\
\hline 10 & Patient & Sheffield, England (1993) \\
\hline 11 & Patient & Bristol, England (1989) \\
\hline 12 & Patient & Bristol, England (1989) \\
\hline 13 & Patient & Rotterdam, The Netherlands (1987) \\
\hline 14 & Patient & Venlo, The Netherlands (1986) \\
\hline 15 & Patient & Dordrecht, The Netherlands (1984) \\
\hline 16 & Patient & Leeds, England (1993) \\
\hline 17 & Patient & Leeds, England (1993) \\
\hline 18 & Patient & Leeds, England (1993) \\
\hline 19 & Patient & Leeds, England (1993) \\
\hline 20 & Patient & Paris, France (1993) \\
\hline 21 & Patient & Paris, France (1993) \\
\hline 22 & Patient & Paris, France (1993) \\
\hline 23 & Patient & CHN, Nottingham, England (1993) \\
\hline 24 & Patient & CHN, Nottingham, England (1985) \\
\hline 25 & Patient & CHN, Nottingham, England (1985) \\
\hline 26 & Patient & CHN, Nottingham, England (1985) \\
\hline 27 & Patient & Cardiff, Wales (1993) \\
\hline 28 & Patient & East Glamorgan, Wales (1993) \\
\hline 29 & Patient & Methyr Tydfil, Wales (1993) \\
\hline
\end{tabular}

UHN, University Hospital Nottingham; CHN, City Hospital Nottingham. 
nate, citrate, glutarate, L-histidine, 4-hydroxybenzoate, L-leucine, levulinate, phenylacetate, L-phenylalanine, phenylpyruvate, protocatechuate, L-tartrate, L-tryptophan and vanillate; growth at $37^{\circ} \mathrm{C}$ and $44^{\circ} \mathrm{C}$; glucose oxidation or fermentation $(\mathrm{O} / \mathrm{F})$; gelatinase production; haemolysis of sheep and human blood; and hydrolysis of pNP- $\beta$-D-xylopyranoside and $\gamma$-L-glutamate-pNA. Tests were performed and assessed as described previously $[14,15]$. Results were scored as positive or negative and then assessed by the DENDRON computer program (Solltech Inc., Oakdale, IA, USA) for Macintosh computers. The DENDRON program computes similarity coefficients between every possible pair of strains and then uses an unweighted pair group method to generate a dendrogram from a matrix of similarity coefficient values. For comparative purposes, all dendrograms produced by the DENDRON program were assessed for grouping at the same $72 \%$ similarity level used for the RAPD-ALFA analysis.

\section{Antibiogram typing}

The method used was based on the disk inhibition zone method described by Horrevorts et al. [16], with the modification that plates were inoculated with a soft agar overlay (Isosensitest Agar $0.7 \% \mathrm{w} / \mathrm{v}$; Oxoid) containing c. $10^{4}$ cells of the strain being tested to give an evenly distributed bacterial lawn. The 15 disks used to test each strain contained the following antibiotics $(\mu \mathrm{g} /$ disk): amikacin (30), ampicillin (10), cefotaxime (30), ceftazidime (30), cefuroxime (30), cephradine (30), chloramphenicol (10), ciprofloxacin (1), gentamicin (10), imipenem (10), kanamycin (30), nalidixic acid (30), rifampicin (5), tetracycline (10) and tobramycin (10). Plates were incubated for $24 \mathrm{~h}$ at $30^{\circ} \mathrm{C}$ and the diameters of the inhibition zones were measured. Similarity coefficients were then calculated and subjected to cluster analysis by the DENDRON program.

\section{Analysis of pulsed-field gel electrophoresis (PFGE) fingerprints}

Intact chromosomal DNA was prepared in agarose plugs by the method of Curran et al. [17] from cultures of Acinetobacter strains grown overnight in Brain Heart Infusion Broth (Oxoid) on an orbital shaker $(150 \mathrm{rpm})$ at $30^{\circ} \mathrm{C}$. PFGE fingerprints were generated by digestion of intact chromosomal DNA with ApaI, and were visualised and photographed as described previously [10]. Fingerprint data were digitised by scanning the photographs (UMAX UC840 Scanner; Leicester Computer Centre). Similarity coefficients were then calculated and subjected to cluster analysis by the DENDRON program.

\section{Analysis of RAPD fingerprints on agarose gels}

Conventional RAPD fingerprints were produced with the following primers: (1) M13 forward and reverse sequencing primers (5'-GTTTTCCCAGTCACGAC-3' and 5'-CAGGAAACAGCTATGAC-3', respectively) used together as described by Vila et al. [18]; (2) DAF4 (see above) used as described by Wiedmann-AlAhmad et al. [13]; and (3) AP3 (5'-TCACGATGCA$\left.3^{\prime}\right)$ used as described by Barbut et al. [19]. Amplification products were visualised and photographed after separation on agarose $1.5 \% \mathrm{w} / \mathrm{v}$ gels [18]. Fingerprint data were digitised and analysed by the DENDRON program as described above.

\section{Results \\ RAPD-ALFA}

Figs 1 and 2 show the dendrograms produced by cluster analysis by GelCompar of the complex RAPD-ALFA fluorogram data obtained with the M13 and DAF4 primers, respectively.

The M13 primer (Fig. 1) yielded four clusters at the $72 \%$ similarity level [7] (cluster 1: strains nos 4, 7, 5, $6,24,25,23,26,27,28,29$ and 1; cluster 2: strains nos 14, 15, 21 and 10; cluster 3: strains nos. 13, 20, 16, 17 and 22; and cluster 4: strains nos 11 and 12, with strain no. 18 linked at a level just below 70\%). RAPD-ALFA with the M13 primer linked the geographically related strains from Nottingham and Wales within one cluster at the $72 \%$ similarity level, although strain no. 1 - which was isolated in 1982 separately from the other strains - fell only just within the cluster. Subtler relationships were discernible within the cluster; thus strains nos $4,7,5$ and 6 from Nottingham University Hospital (UHN) and strains nos 24, 25, 23 and 26 from Nottingham City Hospital (CHN) formed two smaller clusters at the $95 \%$ similarity level. The three strains from Wales (strains nos 27, 2829 ) seemed to be related closely to the CHN strains ( $96 \%$ similarity).

RAPD-ALFA with the DAF4 primer (Fig. 2) also linked the strains from Nottingham and Wales, with the exception of the older strain no. 1 . The only other clusters recognised at the $72 \%$ similarity level were between strains nos 16 and $17 ; 14$ and $15 ; 13$ and 20; 11,12 and 18 (see Discussion).

\section{Biotyping}

Of the 27 tests included in the analysis, 14 gave identical results for all 25 strains, whereas the remaining 13 tests (growth with phenylacetate, citrate, phenylpyruvate, vanillate, levulinate, citraconate, Lphenylalanine, 4-hydroxybenzoate and L-tartrate; glucose $\mathrm{O} / \mathrm{F}$; gelatinase production; hydrolysis of $\mathrm{pNP}-\beta$ $\mathrm{D}$-xylopyranoside; growth at $44^{\circ} \mathrm{C}$ ) identified only minor differences between some of the strains. Consequently, after cluster analysis by the DENDRON program (Fig. 3), all of the strains formed a single 

30
40
50
60
$70 \quad 80$
90
100

20

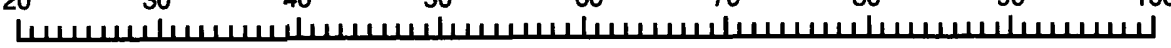

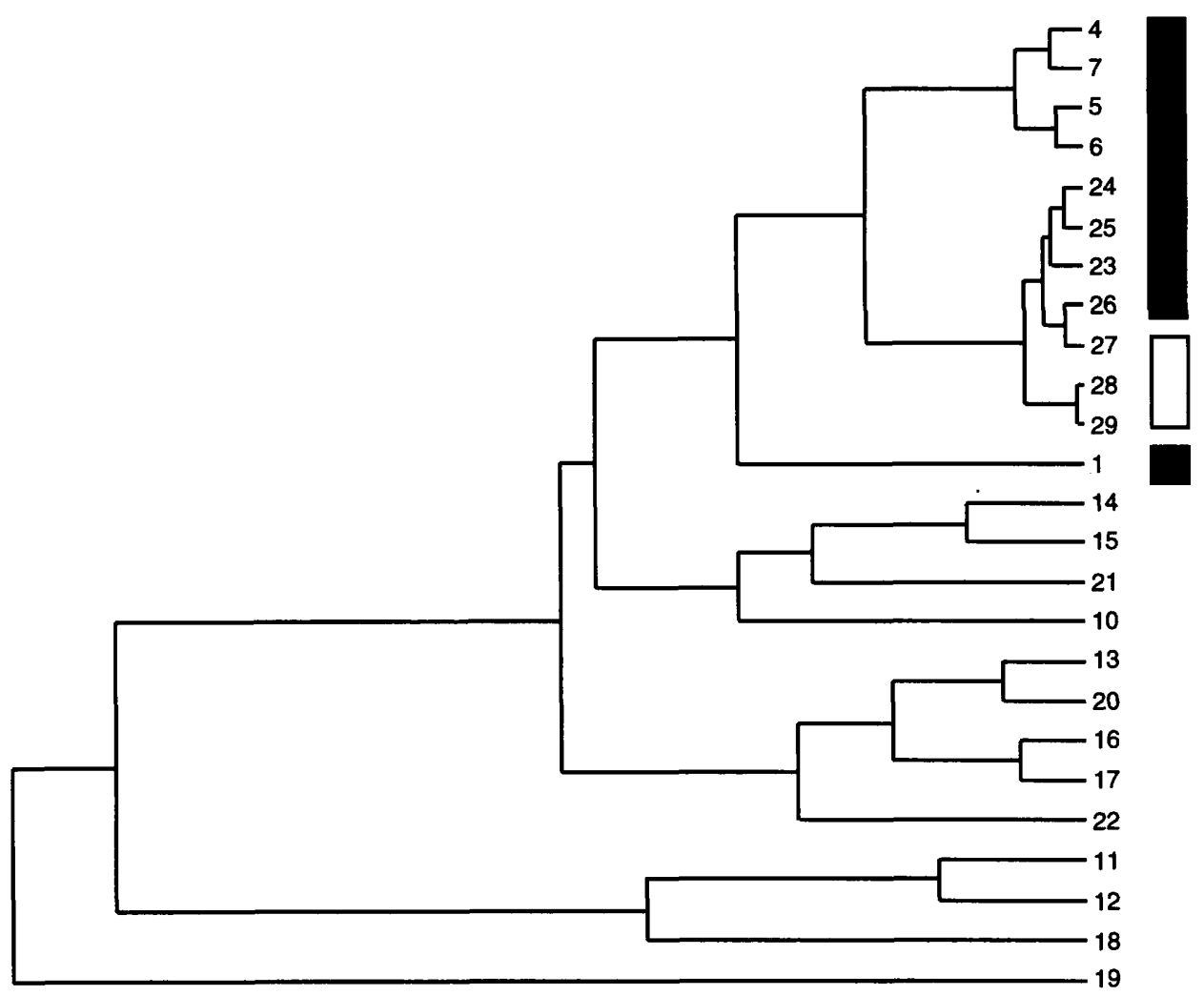

Fig. 1. Dendrogram obtained by cluster analysis of RAPD-ALFA (M13 primer) results with the GelCompar program ( $\square$, Nottingham strains; $\square$, Wales strains). Percentage similarities calculated by the GelCompar program are shown above the dendrogram.

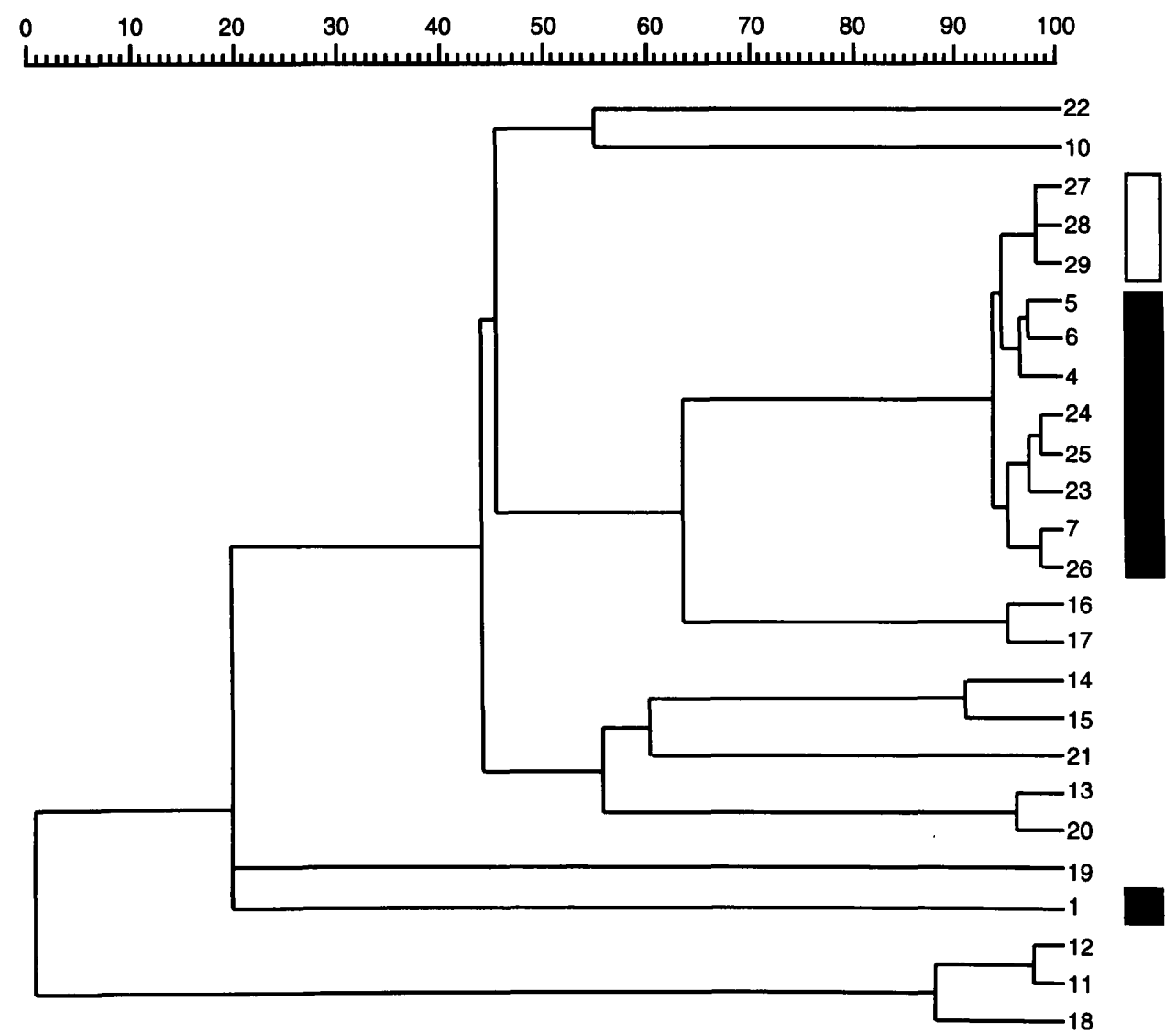

Fig. 2. Dendrogram obtained by cluster analysis of RAPD-ALFA (DAF4 primer) results with the GelCompar program ( $\square$, Nottingham strains; $\square$, Wales strains). Percentage similarities calculated by the GelCompar program are shown above the dendrogram. 


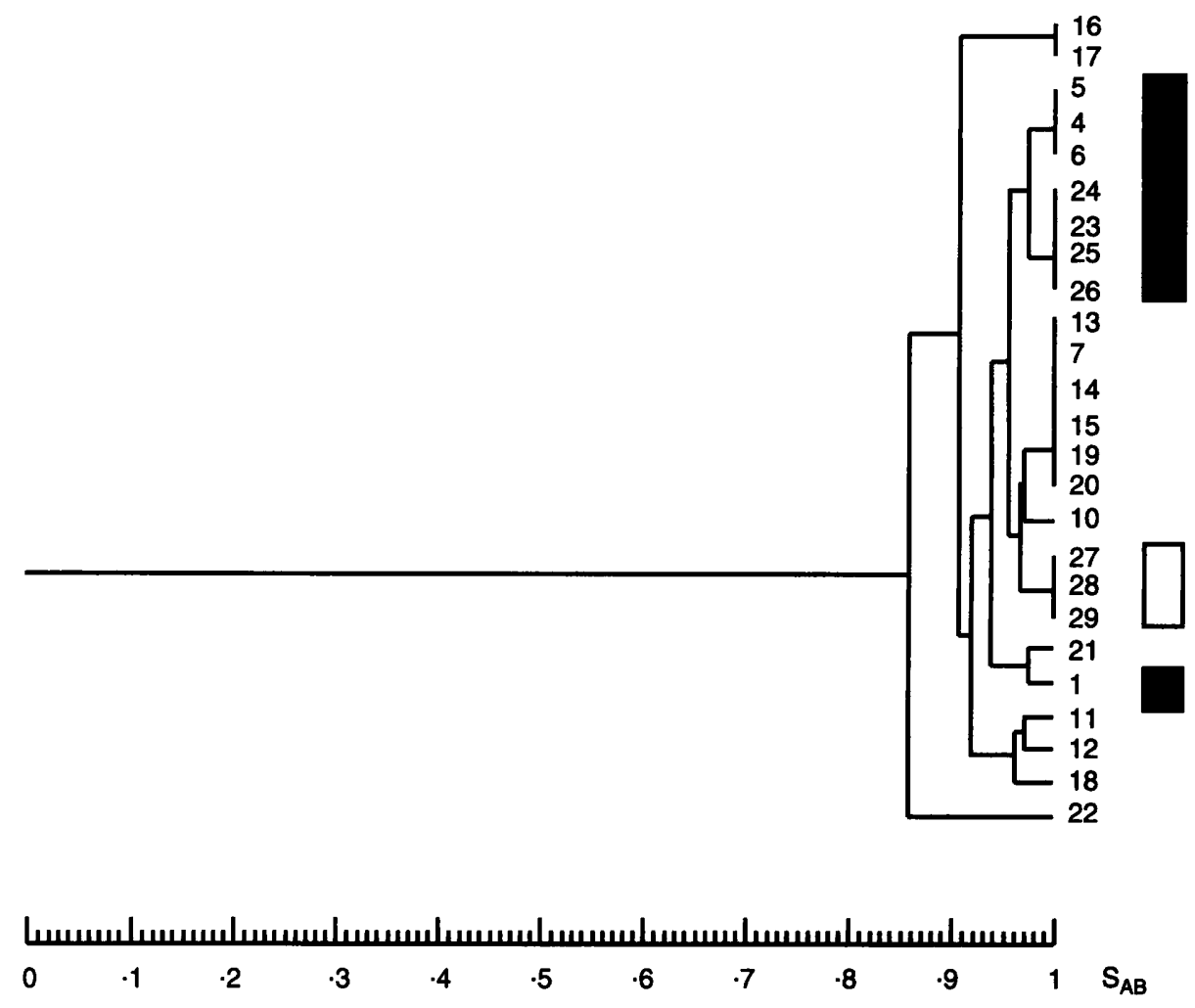

Fig. 3. Dendrogram obtained by cluster analysis of biotyping results with the DENDRON program ( $\square$, Nottingham strains; $\square$, Wales strains). Similarity coefficients (equivalent to percentage similarities divided by 100) calculated by the DENDRON program are shown below the dendrogram.

cluster with $85 \%$ similarity, although the Nottingham strains (with the exception of strain no. 1) and the strains from Wales were linked closely together within the single large cluster. Although growth at $44^{\circ} \mathrm{C}$ is a characteristic normally associated with $A$. baumannii, three strains (nos 11,12 and 18) failed to grow at this temperature (see Discussion).

\section{Antibiogram typing}

The use of inhibition zone diameters meant that only one of the antibiotics included in the analysis (chloramphenicol) gave identical results for all 25 strains tested. Fig. 4 demonstrates that two major clusters were obtained by antibiogram typing at the $72 \%$ similarity level. The three strains from Wales were grouped together fairly tightly within one cluster. Seven of the Nottingham strains were contained within the same cluster, but strains nos 1 and 25 clustered separately.

\section{Analysis of PFGE fingerprints}

The intact chromosomal DNA extracted from one strain (no. 16) was not cut by restriction endonuclease $A p a \mathrm{I}$. The other 24 strains yielded complex Apa I fingerprint patterns, with 12-20 DNA bands being visualised for each strain. None of the bands obtained was common to all 25 strains. The dendrogram obtained by cluster analysis (Fig. 5) shows that the UHN strains (nos 1, 4, 5, 6 and 7) formed a single cluster - with all but strain no. 1 having $72 \%$ similarity - as did the three strains from Wales (nos 27, 28 and 29). The strains from CHN (nos 23, 24, 25 and 26) formed part of two separate clusters, both of which were unlinked to the UHN strain cluster.

\section{Analysis of RAPD fingerprints on agarose gels}

M13 forward and reverse sequencing primers (used together as recommended by Vila et al. [18]) yielded quite extensive strain discrimination (Fig. 6), with 1-12 amplification products (size range $150-1500 \mathrm{bp}$ ) visualised for each strain. None of the amplification products was common to all 25 strains. The three strains from Wales (nos 27, 28 and 29) appeared to be identical, and the CHN strains (nos 23, 24, 25 and 26) were also linked - albeit only at a non-significant similarity level of $55 \%$. Three of the five UHN strains (nos 4, 5 and 6) appeared to be identical and strains nos 1 and 7 were within the same group at a similarity level of $55 \%$.

The DAF4 and AP3 primers both yielded fewer amplification products (1-7 for each strain; size ranges $150-1500 \mathrm{bp}$ and $150-1000 \mathrm{bp}$, respectively), and the respective dendrograms (Figs 7 and 8) indicated that all the strains from Nottingham and 


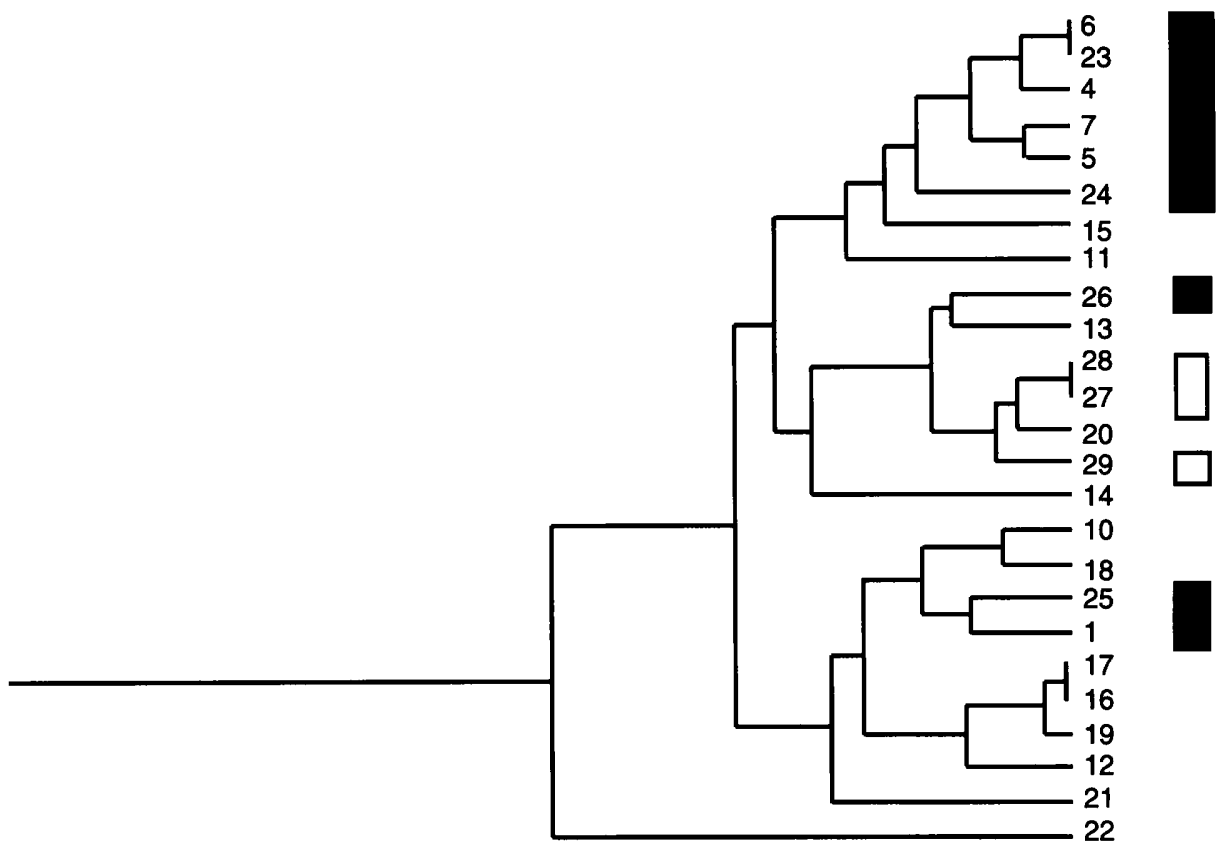

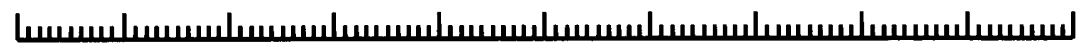

$\begin{array}{llllllllllll}0 & .1 & .2 & .3 & .4 & .5 & .6 & .7 & .8 & .9 & 1 & \mathrm{~S}_{\mathrm{AB}}\end{array}$

Fig. 4. Dendrogram obtained by cluster analysis of antibiogram typing results with the DENDRON program ( $\boldsymbol{\square}$, Nottingham strains; $\square$, Wales strains). Similarity coefficients (equivalent to percentage similarities divided by 100) calculated by the DENDRON program are shown below the dendrogram.

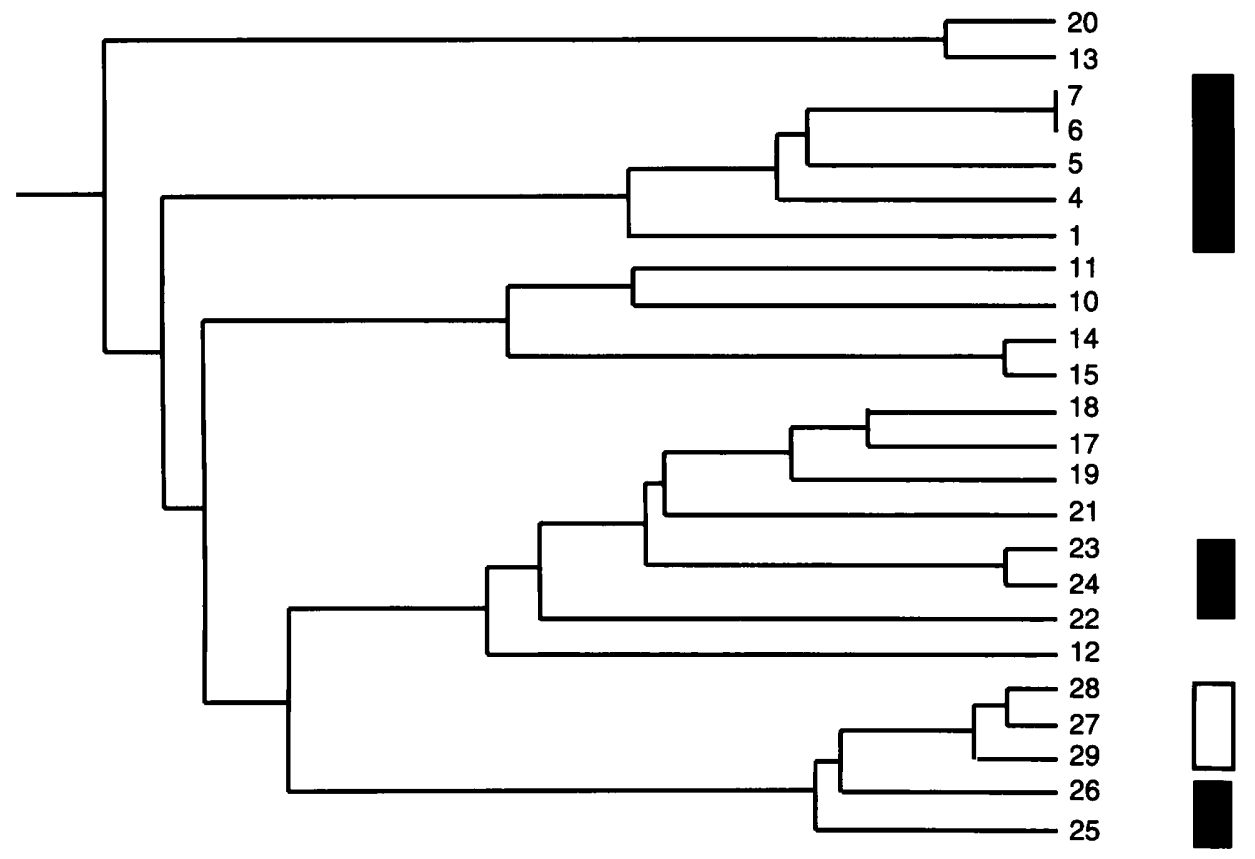

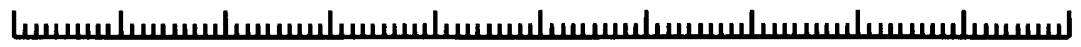

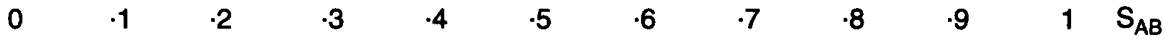

Fig. 5. Dendrogram obtained by cluster analysis of PFGE fingerprinting results with the DENDRON program ( $\boldsymbol{\square}$, Nottingham strains; $\square$, Wales strains). Similarity coefficients (equivalent to percentage similarities divided by 100) calculated by the DENDRON program are shown below the dendrogram. 


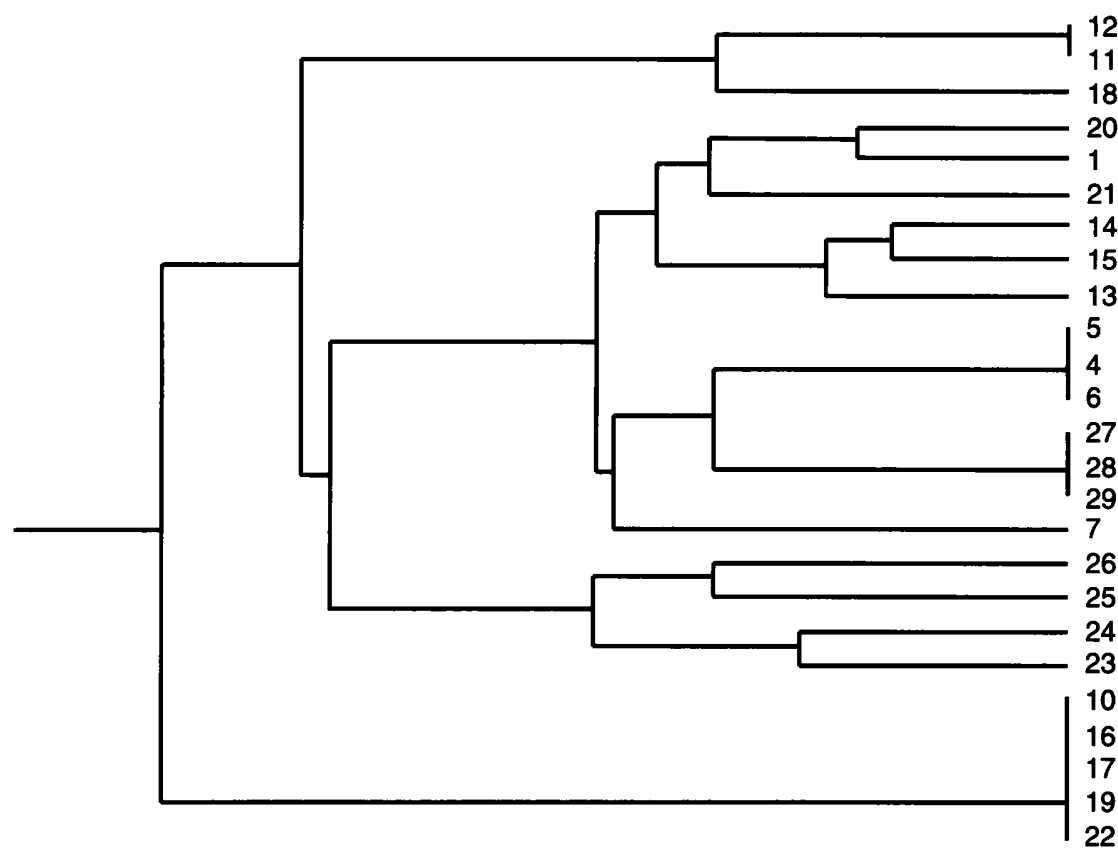

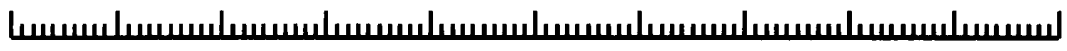

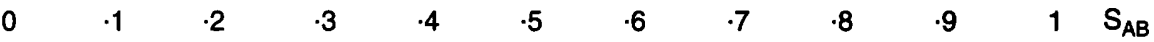

Fig. 6. Dendrogram obtained by cluster analysis of RAPD (M13 forward and reverse primers used together) results with the DENDRON program ( $\square$, Nottingham strains; $\square$, Wales strains). Similarity coefficients (equivalent to percentage similarities divided by 100 ) calculated by the DENDRON program are shown below the dendrogram.
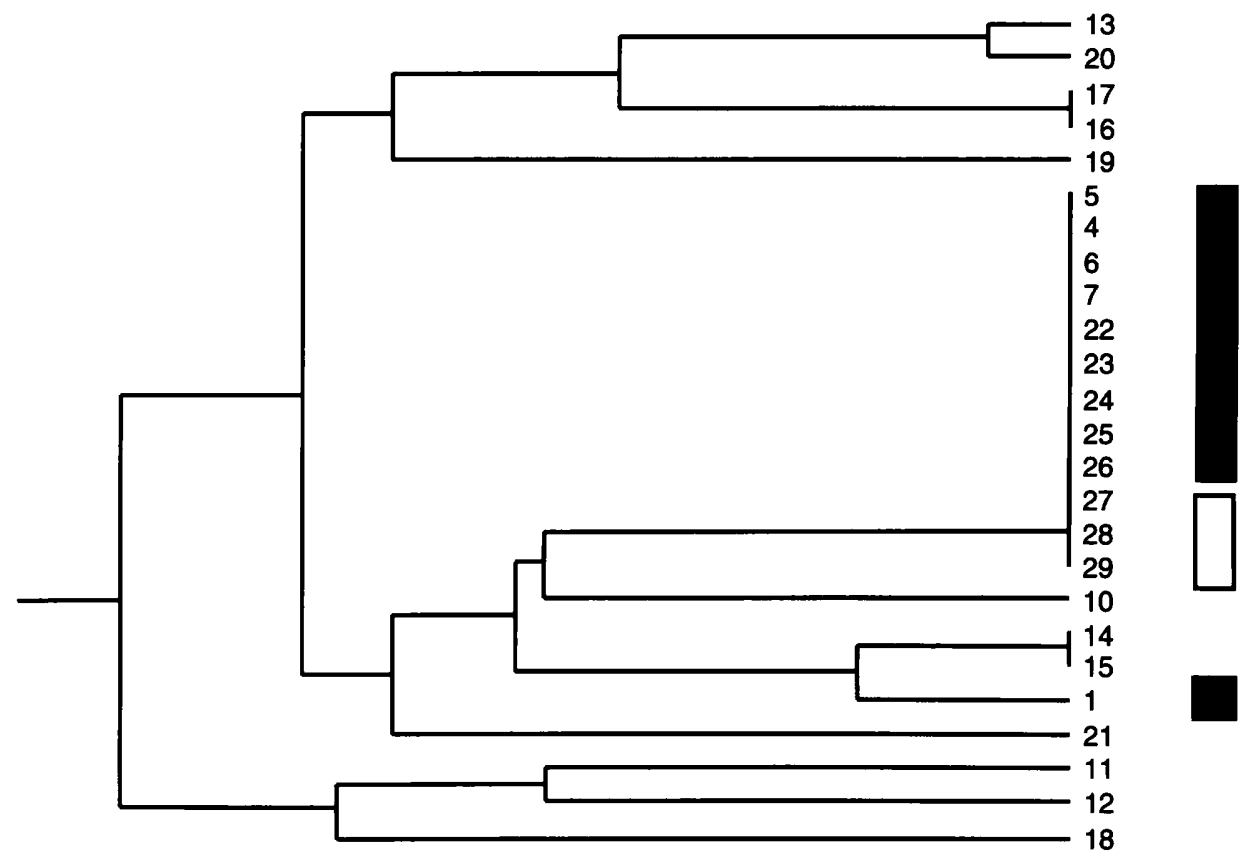

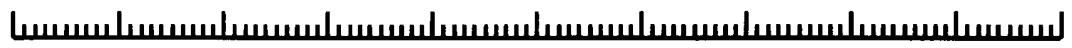

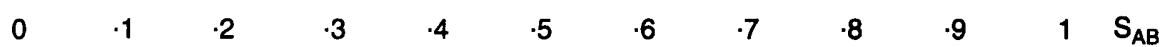

Fig. 7. Dendrogram obtained by cluster analysis of RAPD (DAF4 primer) results with the DENDRON program ( $\boldsymbol{\square}$, Nottingham strains; $\square$, Wales strains). Similarity coefficients (equivalent to percentage similarities divided by 100) calculated by the DENDRON program are shown below the dendrogram. 


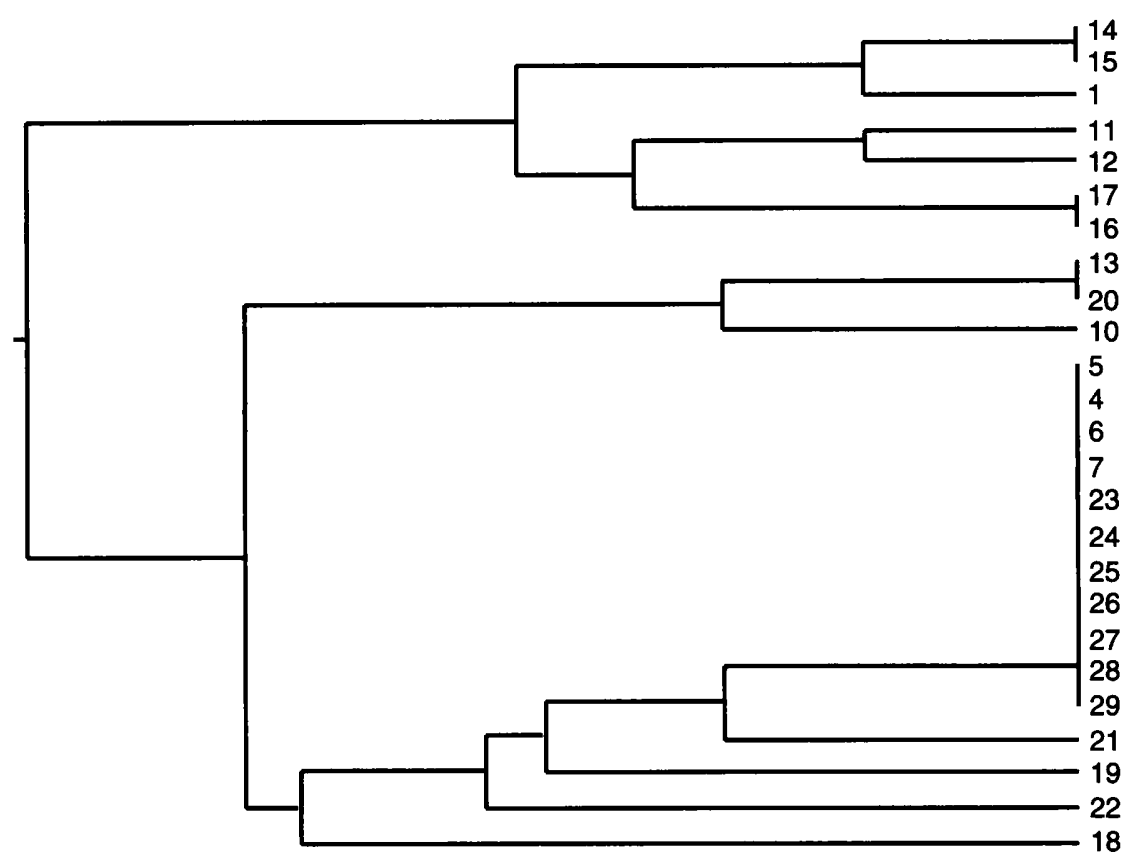

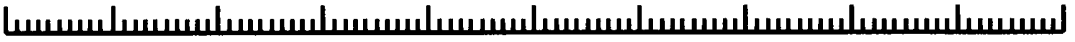

$\begin{array}{llllllllllll}0 & .1 & .2 & .3 & .4 & .5 & .6 & .7 & .8 & .9 & 1 & S_{A B}\end{array}$

Fig. 8. Dendrogram obtained by cluster analysis of RAPD (AP3 primer) results with the DENDRON program ( $\square$, Nottingham strains; $\square$, Wales strains). Similarity coefficients (equivalent to percentage similarities divided by 100) calculated by the DENDRON program are shown below the dendrogram.

Wales - with the exception of the 1982 isolate (strain no. 1) - were identical. Again, no amplification products were common to all 25 strains.

\section{Overall comparison of RAPD-ALFA with other methods}

Table 2 compares the number of geographically related strains that were grouped at the $72 \%$ similarity level by the five computer-assisted typing strategies, as well as the total number of groups identified by each method. Deliberate use was made in this study of two different software packages and clustering algorithms. Neither software package enabled an internal calculation of the topological robustness of the dendrograms generated, but it was possible to examine the overall robustness of the groupings by omitting one or more strains in turn from the DENDRON analysis. When this was done, the

Table 2. Numbers of geographically linked strains grouped at the $72 \%$ similarity level and total number of groups obtained by means of the different computer-assisted typing strategies investigated

\begin{tabular}{|c|c|c|c|c|c|c|c|c|}
\hline \multirow{3}{*}{$\begin{array}{l}\text { Geographically } \\
\text { linked strain } \\
\text { numbers }\end{array}$} & \multicolumn{8}{|c|}{ Number of strains linked at $72 \%$ similarity by } \\
\hline & \multirow[t]{2}{*}{ Biotyping } & \multirow[t]{2}{*}{ Antibiograms } & \multirow[t]{2}{*}{ PFGE } & \multicolumn{2}{|c|}{ RAPD-ALFA } & \multicolumn{3}{|c|}{ RAPD } \\
\hline & & & & DAF4 & M13 & DAF4 & M13† & AP3 \\
\hline $\begin{array}{l}\text { Nottingham } \\
\quad 1,4,5,6,7,23,24,25,26\end{array}$ & 9 & $7 ; 2$ & $5 ; 2 ; 2$ & 8 & 9 & 8 & $3 ; 2$ & 8 \\
\hline Wales $27,28,29$ & 3 & 3 & 3 & 3 & 3 & 3 & 3 & 3 \\
\hline $\begin{array}{l}\text { Bristol } \\
\quad 11,12\end{array}$ & 2 & $x$ & $x$ & 2 & 2 & $x$ & 2 & 2 \\
\hline $\begin{array}{l}\text { Leeds } \\
\qquad 16,17,18,19\end{array}$ & 4 & 4 & $3^{*}$ & 2 & 2 & 2 & 3 & 2 \\
\hline $\begin{array}{l}\text { Paris } \\
20,21,22 \\
\text { The Netherlands }\end{array}$ & 3 & $x$ & $x$ & $x$ & 2 & $x$ & $x$ & $x$ \\
\hline $13,14,15$ & 3 & 3 & 2 & 2 & 2 & 2 & 3 & 2 \\
\hline $\begin{array}{l}\text { Total number of } \\
\text { groups obtained }\end{array}$ & 1 & 3 & 12 & 10 & 6 & 10 & 12 & 10 \\
\hline
\end{tabular}


order in which the strains were presented in the dendrograms was found to vary, but crucially the strain groupings obtained by the various methods for the residual strains were unaltered from those obtained originally for all 25 strains by either program.

\section{Discussion}

DNA fingerprinting methods have become the most commonly used approach for assessing the genetic relatedness of micro-organisms in epidemiological studies. However, before using a particular strategy, it is important to ensure that the method distinguishes unrelated strains, is capable of identifying the same strain in separate samples, and reflects genetic relatedness between epidemiologically linked strains. If only a few strains are being compared, and the associated fingerprints are relatively simple, then visual comparison of pattern differences may be sufficient to assess the degree of relatedness. However, at present there is no general consensus as to the number of 'differences' (i.e., changes in fingerprint pattern) required for two strains to be considered unrelated. For more complex fingerprints, and in cases where strains from different geographical locations are being compared over significant time periods, a computer-assisted strategy is required that enables the formation of a database of fingerprint patterns. Such strategies also enable cluster analysis - leading to the construction of dendrograms showing relationships between strains - and the allocation of a percentage similarity level required to define clusters of related strains.

The work reported here was not concerned with epidemiological relationships amongst $A$. baumannii isolates per se, but aimed to assess the groupings suggested by the novel RAPD-ALFA method [7] in comparison with those suggested by two DNA fingerprinting and two phenotypic typing strategies. Deliberate use was made of a different software package and clustering algorithm for analysing the data generated by the alternative methods, and - as there is no consensus - the $72 \%$ similarity level shown previously [7] to distinguish unambiguously between unrelated strains of $A$. baumannii by RAPDALFA was chosen for the comparative grouping purposes to assess the groups generated by the various methods.

RAPD-ALFA, used with either the M13 or DAF4 primer, successfully clustered the strains from Nottingham and Wales, with the exception of strain no. 1 (isolated in 1982). Minor clusters from other geographical locations were also recognised and, with the partial exception of PFGE analysis (see below), the same clusters were, in general, also recognised by the other methods investigated (Table 2).

Of the two 'phenotypic' strategies examined, biotyping provided the least discrimination, with all 25 strains being grouped within a single cluster at the $72 \%$ similarity level (Fig. 3). Although known geographical links (e.g., the Nottingham and Wales strains) were recognised at a higher level of similarity, discrimination from unrelated strains relied on very small differences within the overall biotype. Strain no. 1 (isolated in 1982) was again separated from the more recent Nottingham isolates, perhaps reflecting some minor evolutionary changes in the predominant endemic Nottingham strain [10] during the passage of time. Although growth at $44^{\circ} \mathrm{C}$ is a characteristic normally associated with $A$. baumannii [15], three strains (nos 11, 12 and 18) failed to grow at this temperature. These strains also formed a cluster when examined by RAPD-ALFA (Figs 1 and 2) and by RAPD with two of the three primers used (Figs 6 and 7), and may, therefore, be representatives of the closely related Acinetobacter genospecies 3 rather than $A$. baumannii $[15,20]$.

Antibiograms of strains are often expressed in terms of 'resistant' or 'sensitive' depending on the breakpoints used. Such an approach has the effect of 'smoothing out' subtler differences that may exist between strains. The alternative approach used in this study, in which inhibition zone sizes were measured, makes full use of all the available information [20] and is being used in an increasing number of typing studies with Acinetobacter spp. [16,21,22]. It should be noted that changes in susceptibility to several related antibiotics (e.g., the aminoglycosides) may be caused by a single genetic event, although the analysis treats all results with equal weight. However, the same caution also applies to the various DNA fingerprinting methods discussed below, in that some individual genetic events (e.g., a spontaneous mutation) will change only one restriction site, whereas others (e.g., a transposon insertion) may change several. Use of antibiograms in the present study provided good discrimination (Fig. 4) and successfully identified the cluster of strains from Wales, but discriminated between some of the Nottingham strains, probably reflecting the acquisition or loss of antibiotic resistance determinants. Antibiotic resistance is often the first indication to a diagnostic laboratory that an outbreak of infections caused by a single organism is occurring, but antibiogram typing results should be interpreted with caution, as unrelated strains may exhibit the same antibiogram and changes in susceptibility may occur during endemic episodes [20].

Of the two additional DNA fingerprinting methods, PFGE analysis following restriction endonuclease digestion with ApaI yielded complex fingerprint patterns, a deeper clonal 'structure' and greater discrimination of the strains than all of the other typing approaches examined, including RAPD-ALFA (Table 2). This is not surprising because PFGE examines the entire genome for restriction fragment 
length polymorphisms and may, therefore, be the most reliable indicator of close relatedness. However, although the strains from Wales and the recent UHN strains were linked (in separate clusters), the CHN strains were split into two separate clusters and were separated from the UHN strains, despite all the other typing methods indicating that the strains from the two Nottingham hospitals were related. This suggests that analysis of $A$. baumannii ApaI PFGE fingerprints may be too sensitive for long-term monitoring and comparisons of endemic episodes of infection during which genetic 'drift' may occur.

Analysis of the RAPD fingerprints on agarose gels provided an almost direct comparison with the automated RAPD-ALFA analysis on high-speed sequencing gels. The clusters obtained with the combined M13 forward and reverse sequencing primers were similar to those obtained by RAPDALFA, although more groups were seen at the $72 \%$ similarity level (Table 2), perhaps reflecting the fact that only a single M13 primer was used in the RAPDALFA experiments. The number of groups obtained with the DAF4 primer was identical by both RAPD and RAPD-ALFA. The overall grouping conclusions obtained by analysis of RAPD fingerprints on agarose gels were virtually identical to those obtained by RAPD-ALFA.

In conclusion, preliminary work with isolates of $A$. baumannii has indicated previously that the novel RAPD-ALFA method has significant advantages compared with other fingerprinting strategies [7]. The work described here, with a defined panel of isolates from different geographical locations, has confirmed that RAPD-ALFA yields an appropriate level of strain discrimination for future epidemiological studies, and that the relationships suggested by cluster analysis of RAPD-ALFA data are essentially similar to those suggested by other methods employing a different software package and clustering algorithm. In the absence of a recognised 'outbreak' of infection, transmission of a single $A$. baumannii strain between a small number of patients will often go undetected unless fingerprint patterns are analysed routinely and compared with a database. The question of cost effectiveness remains to be addressed, but the technique of RAPD-ALFA appears to offer the possibility of routine on-line molecular identification and typing of isolates of a broad range of bacterial species from particular hospital wards or units (e.g., intensive care units), and could, therefore, play a key role in the early recognition and prevention of outbreaks of infection.

We thank Professor E. Bergogne-Bérézin, Dr L. Dijkshoorn, Professor P. Hawkey, Dr I. K. Hosein and Dr S. Partridge for the generous gift of strains. We are also indebted to Trent Regional Health Authority for their financial support of this work. K. J. T. thanks the British Council for a travel grant awarded under the Anglo-German Academic Research Collaboration Programme.

\section{References}

1. Welsh J, McClelland M. Fingerprinting genomes using PCR with arbitrary primers. Nucleic Acids Res 1990; 18: 72137218.

2. Maslow JN, Mulligan ME, Arbeit RD. Molecular epidemiology: application of contemporary techniques to the typing of microorganisms. Clin Infect Dis 1993; 17: 153-164.

3. Caetano-Anollés G, Bassam BJ, Gresshoff PM. DNA amplification fingerprinting using very short arbitrary oligonucleotide primers. Biotechnology 1991; 9: 553-557.

4. Meunier JR, Grimont PAD. Factors affecting reproducibility of random amplified polymorphic DNA fingerprinting. Res Microbiol 1993; 144: 373-379.

5. Towner KJ, Cockayne A. Molecular methods for microbia identification and typing. London, Chapman \& Hall. 1993.

6. Arbeit RD, Maslow JN, Mulligan ME. Polymerase chain reaction-mediated genotyping in microbial epidemiology: reply. Clin Infect Dis 1994; 18: 1018-1019.

7. Grundmann H, Schneider Ch, Tichy HV et al. Automated laser fluorescence analysis of randomly amplified polymorphic DNA: a rapid method for investigating nosocomial transmission of Acinetobacter baumannii. $J$ Med Microbiol 1995; 43 446-451.

8. Voss H, Stegemann J, Schwager C et al. High-speed automated DNA fragment analysis for genome mapping by restriction fingerprinting. Methods Mol Cell Biol 1992; 3: 77-82.

9. Bergogne-Bérézin E, Joly-Guillou ML, Towner KJ (eds). Acinetobacter: microbiology, epidemiology, infections, management. Boca Raton, CRC Press. 1995.

10. Crowe M, Towner KJ, Humphreys H. Clinical and epidemiological features of an outbreak of acinetobacter infection in an intensive therapy unit. $J$ Med Microbiol 1995; 43: 55-62.

11. Bergogne-Bérézin E, Joly-Guillou ML, Vieu JF. Epidemiology of nosocomial infections due to Acinetobacter calcoaceticus. $J$ Hosp Infect 1987; 10: 105-113.

12. Noble WC. Hospital epidemiology of Acinetobacter infection. In: Towner KJ, Bergogne-Bérézin E, Fewson CA (eds) The biology of Acinetobacter: taxonomy, clinical importance, molecular biology, physiology, industrial relevance. New York, Plenum Press. 1991: 53-62.

13. Wiedmann-al-Ahmad M, Tichy HV, Schön G. Characterization of Acinetobacter type strains and isolates obtained from wastewater treatment plants by PCR fingerprinting. Appl Environ Microbiol 1994; 60: 4066-4071.

14. Bouvet PJM, Grimont PAD. Identification and biotyping of clinical isolates of Acinetobacter. Ann Inst Pasteur Microbiol 1987; 138: 569-578.

15. Kämpfer $P$, Tjernberg I, Ursing J. Numerical classification and identification of Acinetobacter genomic species. $J$ Appl Bacteriol 1993; 75: 259-268.

16. Horrevorts A, Bergman K, Kollée L, Breuker I, Tjernberg I, Dijkshoorn L. Clinical and epidemiological investigations of Acinetobacter genomospecies 3 in a neonatal intensive care unit. J Clin Microbiol 1995; 33: 1567-1572.

17. Curran R, Hardie KR, Towner KJ. Analysis by pulsed-field gel electrophoresis of insertion mutations in the transferrin-binding system of Haemophilus influenzae type b. J Med Microbiol 1994; 41: 120-126.

18. Vila J, Marcos A, Llovet T, Coll P, Jimenez de Anta T. A comparative study of ribotyping and arbitrarily primed polymerase chain reaction for investigation of hospital outbreaks of Acinetobacter baumannii infection. $J$ Med Microbiol 1994; 41: 244-249.

19. Barbut F, Mario N, Delmée M, Gozian J, Petit J-C. Genomic fingerprinting of Clostridium difficile isolates by using a random amplified polymorphic DNA (RAPD) assay. FEMS Microbiol Lett 1993; 114: 161-166.

20. Dijkshoorn L. Acinetobacter - microbiology. In: BergogneBérézin E, Joly-Guillou ML, Towner KJ (eds) Acinetobacter: microbiology, epidemiology, infections, management. Boca Raton, CRC Press. 1995: 37-69.

21. Dijkshoorn L, Aucken HM, Gerner-Smidt P, Kaufmann ME, Ursing J, Pitt TL. Correlation of typing methods for Acinetobacter isolates from hospital outbreaks. J Clin Microbiol 1993; 31: 702-705.

22. Weernink A, Severin WPJ, Tjernberg I, Dijkshoorn L. Pillows, an unexpected source of Acinetobacter. J Hosp Infect 1995; 29: 189-199. 